\title{
Massage du sinus carotidien
}

\section{Carotid Sinus Massage}

\section{H. Lu $\cdot$ M. Pasquier $\cdot$ H. Lu}

Reçu le 7 août 2018; accepté le 8 septembre 2018

(C) SFMU et Lavoisier SAS 2018

Résumé Le massage du sinus carotidien (MSC) est un geste simple, utilisé en médecine d'urgence à visées diagnostique et/ou thérapeutique. Les indications principales au MSC sont l'évaluation des syncopes chez les patients de plus de 40 ans et le traitement de première ligne des tachycardies paroxystiques supraventriculaires bien tolérées hémodynamiquement. Les contre-indications incluent un antécédent d'accident vasculaire cérébral ou d'accident ischémique transitoire dans les trois mois qui précèdent ainsi que la présence d'une sténose carotidienne significative ou d'un souffle carotidien. Dans le bilan de la syncope, le MSC permet de diagnostiquer une hypersensibilité du sinus carotidien. Pour les tachycardies supraventriculaires, il permet de préciser le type de tachycardie et, dans certains cas, d'obtenir une restauration d'un rythme sinusal. Les rares complications sont liées au risque de provoquer une embolie cérébrale.

\section{Mots clés Sinus carotidien $\cdot$ Syncope $\cdot$ Tachycardie}

\begin{abstract}
Carotid sinus massage is a simple procedure used in emergency medicine for diagnostic and/ or therapeutic purposes. The main indications for carotid sinus massage are the evaluation of syncope in patients over 40 years of age; it is also the first-line treatment of hemodynamically stable supraventricular paroxysmal tachycardia. Contraindi-
\end{abstract}

\section{H. Lu}

Service des urgences adultes,

centre hospitalier universitaire Antoine-Béclère,

Assistance publique-hôpitaux de Paris (AP-HP),

157, rue de la Porte-de-Trivaux,

F-92140 Clamart, France

M. Pasquier

Service des urgences,

Centre hospitalier universitaire vaudois (CHUV),

rue du Bugnon 46, CH-1011 Lausanne, Suisse

H. Lu ( $\square)$

Service de médecine interne,

Centre hospitalier universitaire vaudois (CHUV),

rue du Bugnon 46, CH-1011 Lausanne, Suisse

e-mail : henri.lu@chuv.ch cations include a history of stroke or transient ischemic attack within the previous three months, as well as significant carotid artery stenosis or a carotid bruit on auscultation. In the evaluation of syncope, carotid sinus massage can reveal carotid sinus hypersensitivity. In the case of supraventricular tachycardia, it can help to clarify the type of tachycardia and in some cases, obtain a restoration of a sinus rhythm. The rare complications are related to the risk of causing cerebral embolism.

Keywords Carotid sinus $\cdot$ Syncope $\cdot$ Tachycardia

\section{Introduction}

Le massage du sinus carotidien (MSC) est un geste fréquemment réalisé en médecine d'urgence. Ses indications principales sont le dépistage d'une hypersensibilité du sinus carotidien dans le cadre d'un bilan de vertiges ou de syncopes et le diagnostic, voire le traitement des tachycardies paroxystiques. Cet article a pour objectif de faire le point sur les indications, les contre-indications, l'équipement et les techniques nécessaires pour effectuer la procédure en sécurité chez le patient adulte.

\section{Histoire}

L'effet bradycardisant d'une pression exercée sur la bifurcation carotidienne a été mis en évidence par Czermak en 1866. En 1927, Hering montra que la pression sur la bifurcation stimule le sinus carotidien et non le nerf vague, comme supposé auparavant [1]. En 1933, Weiss et Baker prouvèrent qu'une augmentation de la sensibilité du sinus carotidien peut causer des syncopes et proposèrent une classification de l'hypersensibilité du sinus carotidien selon deux critères encore utilisés de nos jours (diminution de la fréquence cardiaque et diminution de la tension artérielle, cf. infra) [2]. L'application clinique du MSC a été revue en détail par Lown et Levine en 1961. Enfin, Levy et al. suggérèrent que les barorécepteurs 
artériels pouvaient jouer un rôle important dans la pathogenèse de certaines arythmies [3].

\section{Physiopathologie}

Le sinus carotidien détecte les modifications de la pression artérielle systémique par le biais de barorécepteurs. Ces derniers, situés au-dessus de la bifurcation carotidienne et au niveau de la crosse aortique, sont sensibles à l'extension des parois vasculaires.

Le système afférent de l'arc réflexe des barorécepteurs est composé du nerf de Hering, localisé au niveau du sinus carotidien, ainsi que du nerf de Cyon, situé au niveau de la crosse aortique. Ces nerfs véhiculent le message nerveux sensitif vers le noyau du tractus solitaire (NTS) bulbaire. Le système efférent est quant à lui composé d'une voie parasympathique médiée par le nerf vague et d'une voie sympathique médiée par la chaîne ganglionnaire sympathique paravertébrale. La figure 1 résume les voies de l'arc réflexe des barorécepteurs.

Chez un sujet au repos, le système des barorécepteurs artériels exerce en permanence un tonus inhibiteur sur les efférences sympathiques et une activation des noyaux bulbaires parasympathiques. En cas d'hypotension artérielle, la baisse de stimulation des barorécepteurs entraîne une diminution de la réponse afférente destinée au NTS. On observe alors une activation de la voie sympathique et une inhibition de la voie parasympathique. Il en résulte une augmentation de l'inotropisme et du chronotropisme cardiaques, une tachycardisation, une vasoconstriction des vaisseaux périphériques. Inversement, en cas d'hypertension artérielle, l'activation des barorécepteurs entraîne une augmentation de la réponse afférente vers le NTS, ce qui stimule la voie parasympathique et provoque une bradycardie et une vasodilatation.

Dans l'hypersensibilité du sinus carotidien, il semble que les manifestations cliniques (lipothymie, syncope) soient provoquées par une réponse bulbaire exagérée face à des stimuli peu importants de la voie afférente [4]. Par ailleurs, toute manipulation du sinus carotidien (massage, dissection carotidienne, etc.) provoque une stimulation des barorécepteurs et donc une prédominance parasympathique induisant bradycardie et hypotension.

\section{Indications}

Le MSC a deux indications principales :

- l'évaluation des syncopes chez les patients de plus de 40 ans, chez qui l'étiologie n'est pas retrouvée après une anamnèse et un examen clinique initiaux $[3,5]$;

- le traitement de première ligne des tachycardies paroxystiques supraventriculaires qui sont hémodynamiquement

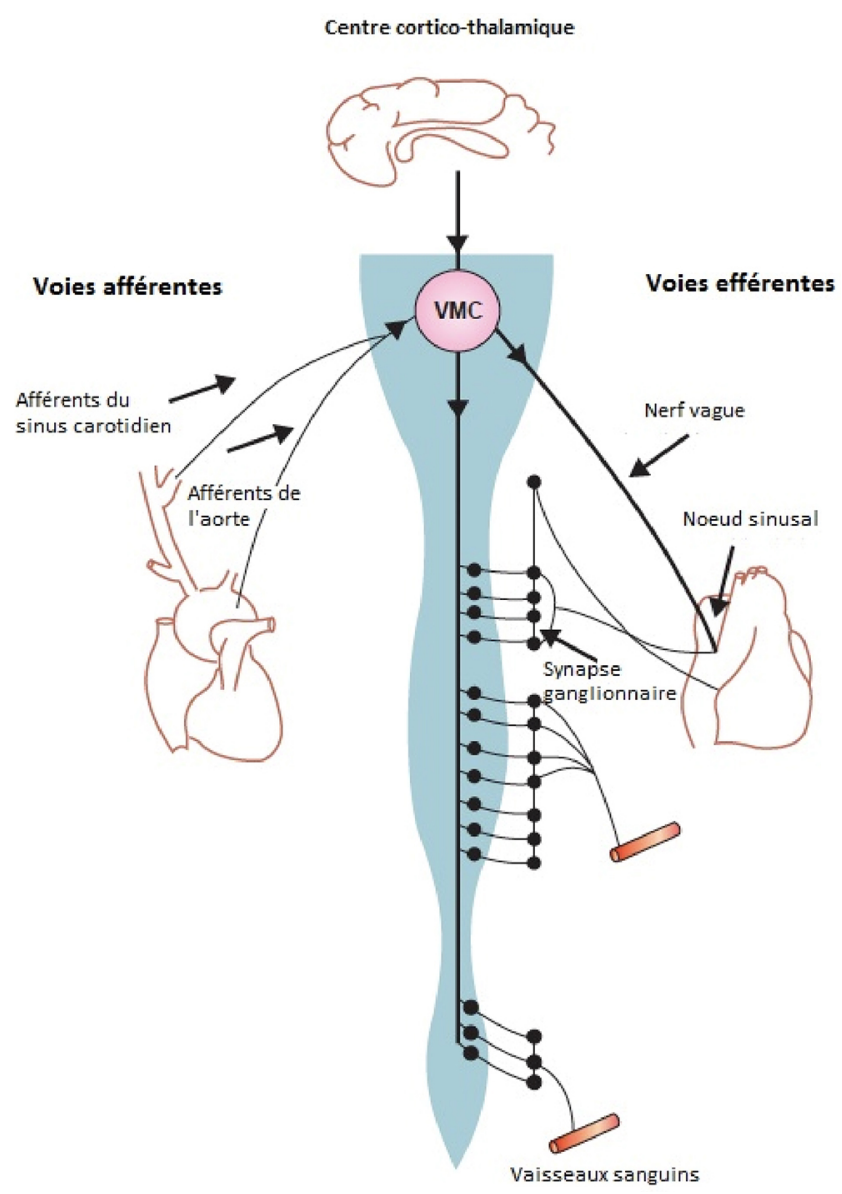

Fig. 1 Schéma des voies afférentes et efférentes de l'arc réflexe des barorécepteurs. Tiré et adapté de [3], publié avec l'aimable autorisation du Dr Wieling et de John Wiley and Sons Inc. (Reprinted with permission, John Wiley and Sons, Inc.)

stables. Dans ce cas particulier, le MSC peut également aider à clarifier le type et l'origine de la tachycardie supraventriculaire [6].

\section{Contre-indications}

Le MSC est contre-indiqué chez les patients à risque de faire un accident vasculaire cérébral (AVC) ou un accident ischémique transitoire (AIT) ou qui présentent une pathologie carotidienne. D'après les recommandations de l'American Heart Association [7], le MSC est contre-indiqué chez les patients ayant :

- un antécédent d'AVC ou d'AIT dans les trois mois qui précèdent ;

- une sténose significative carotidienne ou un souffle carotidien. 
Il faut cependant noter qu'un souffle carotidien n'est pas un indicateur fiable d'une pathologie carotidienne. Ainsi, chez les patients à haut risque d'AVC, ceux ayant une coronaropathie connue ou une artériopathie périphérique, il peut être utile d'effectuer une échographie Doppler des artères carotides par un médecin expérimenté avant d'effectuer un MSC.

Un antécédent de complications au cours d'un MSC passé, un infarctus du myocarde dans les trois mois précédents ou des antécédents d'arythmie ventriculaire sont également des contre-indications $[8,9]$.

\section{Équipement}

L'équipement nécessaire pour effectuer un MSC comprend : un stéthoscope, un moniteur cardiaque capable d'enregistrer le rythme cardiaque de manière continue et de mesurer la pression artérielle, ainsi qu'une machine à électrocardiogramme (ECG). Les médicaments et le matériel nécessaires à une éventuelle réanimation cardiopulmonaire doivent être disponibles. Le patient devrait être équipé d'une voie veineuse périphérique.

\section{Préparation}

Afin d'assurer le bon déroulement du MSC, nous proposons de suivre les étapes suivantes :
- expliquer la procédure au patient. En cas de suspicion d'une hypersensibilité du sinus carotidien, informer le patient que le MSC est susceptible de reproduire une sensation de lipothymie ou de présyncope. L'informer également que le MSC peut créer une gêne ou une douleur au niveau de la zone de pression;

- confirmer l'identité du patient, l'indication du MSC et l'absence de contre-indication ;

- placer le patient en décubitus dorsal strict ;

- déterminer la localisation de l'artère carotide (cf. infra). Ausculter cette dernière pour éliminer un souffle carotidien, comme suit : placer le pavillon du stéthoscope sur l'artère carotide et demander au patient de retenir sa respiration pendant l'auscultation ;

- surveiller la fréquence cardiaque et mesurer la pression artérielle à une fréquence prédéfinie (toutes les deux minutes).

\section{Geste}

Le sinus carotidien est localisé à la bifurcation de l'artère carotide commune, qui se trouve au niveau du cartilage thyroïdien, au-dessous de l'angle de la mandibule et sur le bord médial du muscle sternocléidomastoïdien. Pour le repérer, positionner l'index et le majeur au niveau du cartilage thyroïdien puis les déplacer latéralement jusqu'à ressentir le pouls carotidien (Fig. 2).

Avant d'effectuer le MSC, il convient d'informer le patient que l'on est sur le point d'effectuer le geste, puis de

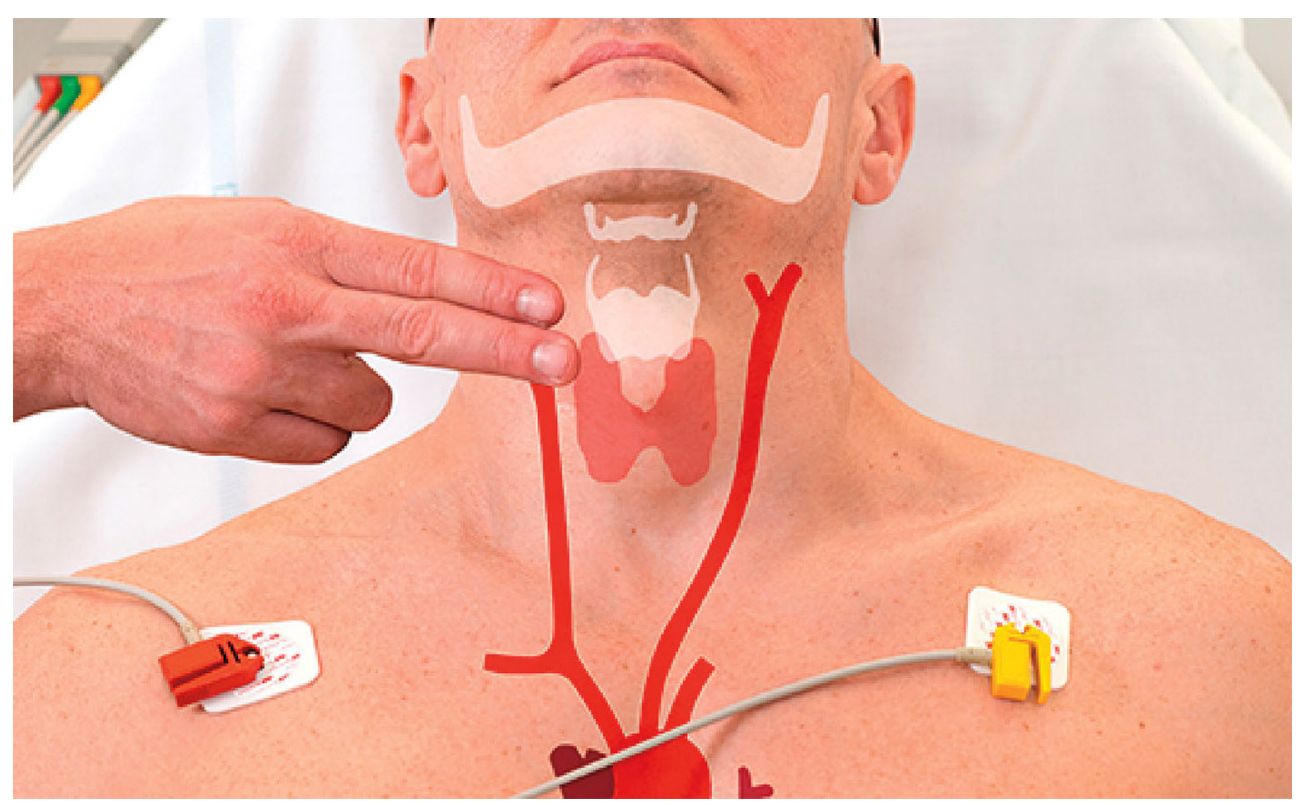

Fig. 2 Localisation du sinus carotidien par rapport au cartilage thyroïdien et à la mandibule D'après Pasquier et al. [6] (Copyright $(C)$ 2017 Massachusetts Medical Society. Reprinted with permission) 
demander à un assistant de débuter l'enregistrement d'un tracé ECG continu. Le massage est effectué en appliquant une pression ferme et constante sur un des deux sinus carotidiens. Il ne faut pas appuyer de manière excessivement forte, afin de ne pas occlure l'artère. Il est contre-indiqué de masser les deux sinus simultanément. Une pression constante est recommandée, car elle est plus reproductible qu'une pression intermittente [8]. Il ne faut pas oublier d'informer l'assistant du moment précis où l'on commence à appliquer la pression, pour pouvoir le noter sur le tracé de l'ECG, et d'observer le patient et le moniteur durant la procédure. Le massage doit durer au moins cinq secondes sans dépasser dix secondes.

Si le MSC sur un côté ne produit pas de réponse ni de complication, un MSC peut être réalisé du côté opposé, quelle que soit l'indication du geste, une fois que le patient est prêt et que la fréquence cardiaque et la pression artérielle sont revenues aux valeurs antérieures. Si le MSC est effectué pour une évaluation de syncope, il est recommandé de répéter le test en position debout en utilisant une table basculante [10].

\section{Réponses au MSC}

\section{Bilan de syncope}

Sur le plan rythmique, la réponse physiologique à un MSC est une bradycardisation et un ralentissement de la conduction au niveau du nœud atrioventriculaire. Le MSC peut aussi entraîner une diminution de l'amplitude de l'onde P.
Sur le plan tensionnel, la réponse typique est une chute de tension induite par la diminution du tonus vasculaire [3].

Dans le bilan étiologique d'une syncope, une pause ventriculaire de plus de trois secondes ou plus et/ou une diminution de la pression artérielle systolique de $50 \mathrm{mmHg}$ définissent une hypersensibilité du sinus carotidien (Fig. 3). Le diagnostic est certain si une syncope ou une lipothymie est reproduite pendant ou immédiatement après le massage en présence d'au moins un des deux critères sus-cités.

Il est possible de définir trois types d'hypersensibilité du sinus carotidien selon les réponses rythmique et tensionnelle au MSC :

- la forme cardio-inhibitrice est définie par une pause de trois secondes ou plus sans diminution de la pression artérielle de $50 \mathrm{mmHg}$;

- la forme vasodépressive est définie par une diminution de la pression artérielle de $50 \mathrm{mmHg}$ ou plus sans pause de trois secondes;

- une forme mixte est définie par l'association d'une pause de trois secondes ou plus avec la diminution de la pression artérielle de $50 \mathrm{mmHg}$ ou plus [11].

La caractérisation du type d'hypersensibilité du sinus carotidien est utile non seulement dans le cadre du diagnostic, mais également pour guider le traitement. En effet, seule la forme cardio-inhibitrice répond à un entraînement électrosystolique $[7,12]$.

Dans un bilan de syncope d'étiologie indéterminée, il est recommandé d'effectuer le MSC suite au passage de la position allongée à la position assise, puis debout, le patient

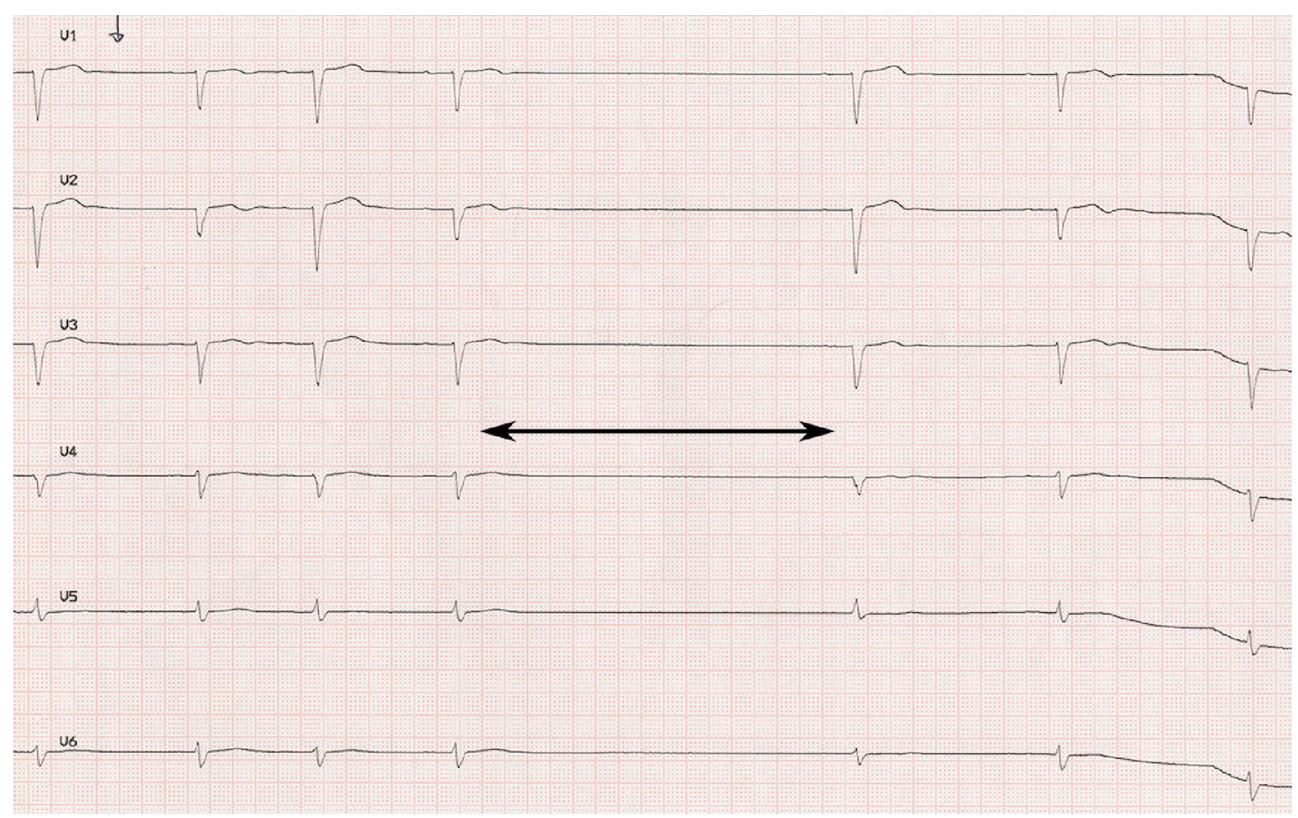

Fig. 3 ECG montrant une pause ventriculaire de 3,5 secondes après un massage du sinus carotidien D'après Pasquier et al. [6] (Copyright (C) 2017 Massachusetts Medical Society. Reprinted with permission) 
(attaché de manière sécurisée afin de prévenir une éventuelle chute) étant positionné sur une table basculante [10]. De cette manière, la sensibilité du test est augmentée, et l'ampleur de la composante vasodépressive peut être évaluée de manière plus aisée. Sous-estimée dans le passé, une composante vasodépressive est en fait présente chez la plupart des patients présentant une réponse cardio-inhibitrice. Déterminer la part vasodépressive du réflexe présente une importance pratique dans le choix du traitement, puisque l'efficacité d'un entraînement électrosystolique risque d'être diminuée [7].

\section{Diagnostic et traitement d'une tachycardie supraventriculaire}

Dans le cadre d'une tachycardie supraventriculaire, les réponses dépendent du type de tachycardie et sont résumées dans le tableau 1. Un retour en rythme sinusal peut être observé en cas de tachycardie jonctionnelle (Fig. 4). La physiopathologie sous-tendant ces réponses relève de la rythmologie et n'est pas l'objet de cette revue, elle a été résumée par Schweitzer et Teichholz [3]. À noter que dans certains cas le MSC peut produire un bloc atrioventriculaire qui peut démasquer une activité atriale sous-jacente, permettant le diagnostic d'un flutter atrial ou d'une fibrillation auriculaire (Fig. 5).

Il est également important de souligner que la manœuvre de Vasalva modifiée est le geste à réaliser en première intention dans le cadre d'une tachycardie supraventriculaire. En effet, il s'agit d'un geste simple, rapide, avec un retour en rythme sinusal dans $43 \%$ des cas, et ne présentant que peu de risques [13].

\section{Complications}

Le risque principal lors de la réalisation d'un MSC est de provoquer le décrochement d'une plaque athéromateuse carotidienne et de provoquer une embolie cérébrale. On estime que $0,5 \%$ des MSC se compliquent d'un AVC ou d'un AIT [14-16]. Le risque d'AVC ou d'AIT peut être diminué en respectant les contre-indications du MSC. Dans de rares cas, un trouble du rythme ventriculaire ou une fibrillation atriale peut être provoqué en réponse à une bradycardie extrême, elle-même causée par le MSC. Les complications mineures du MSC incluent une gêne ou une douleur locale. Des vertiges ou une syncope peuvent aussi apparaitre.

Tableau 1 Réponses usuelles au massage du sinus carotidien dans les tachyarythmies

\begin{tabular}{|lll|}
\hline Arythmie & Réponses classiques & Réponses moins fréquentes \\
\hline Tachycardie sinusale & Ralentissement transitoire de la fréquence & Bloc atrioventriculaire \\
cardiaque & Pas de réponse \\
Tachycardie atriale & Bloc atrioventriculaire & Pas de réponse \\
Flutter atrial & Retour en rythme sinusal & Fibrillation atriale, ralentissement \\
& Bloc atrioventriculaire & de la fréquence cardiaque \\
Fibrillation atriale & & \\
Rythme jonctionnel atrioventriculaire & Bloc atrioventriculaire & \\
accéléré & Ralentissement transitoire de la fréquence & \\
Tachycardie ventriculaire & cardiaque & Ralentissement de la fréquence cardiaque \\
\hline
\end{tabular}

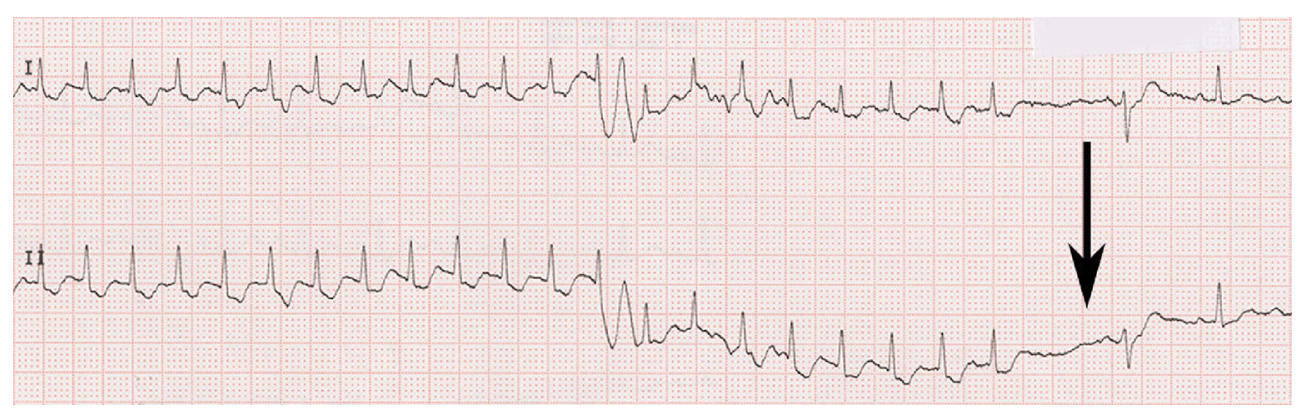

Fig. 4 ECG montrant un retour en rythme sinusal (flèche) d'une tachycardie jonctionnelle après un massage du sinus carotidien D'après Pasquier et al. [6] (Copyright (C) 2017 Massachusetts Medical Society. Reprinted with permission) 


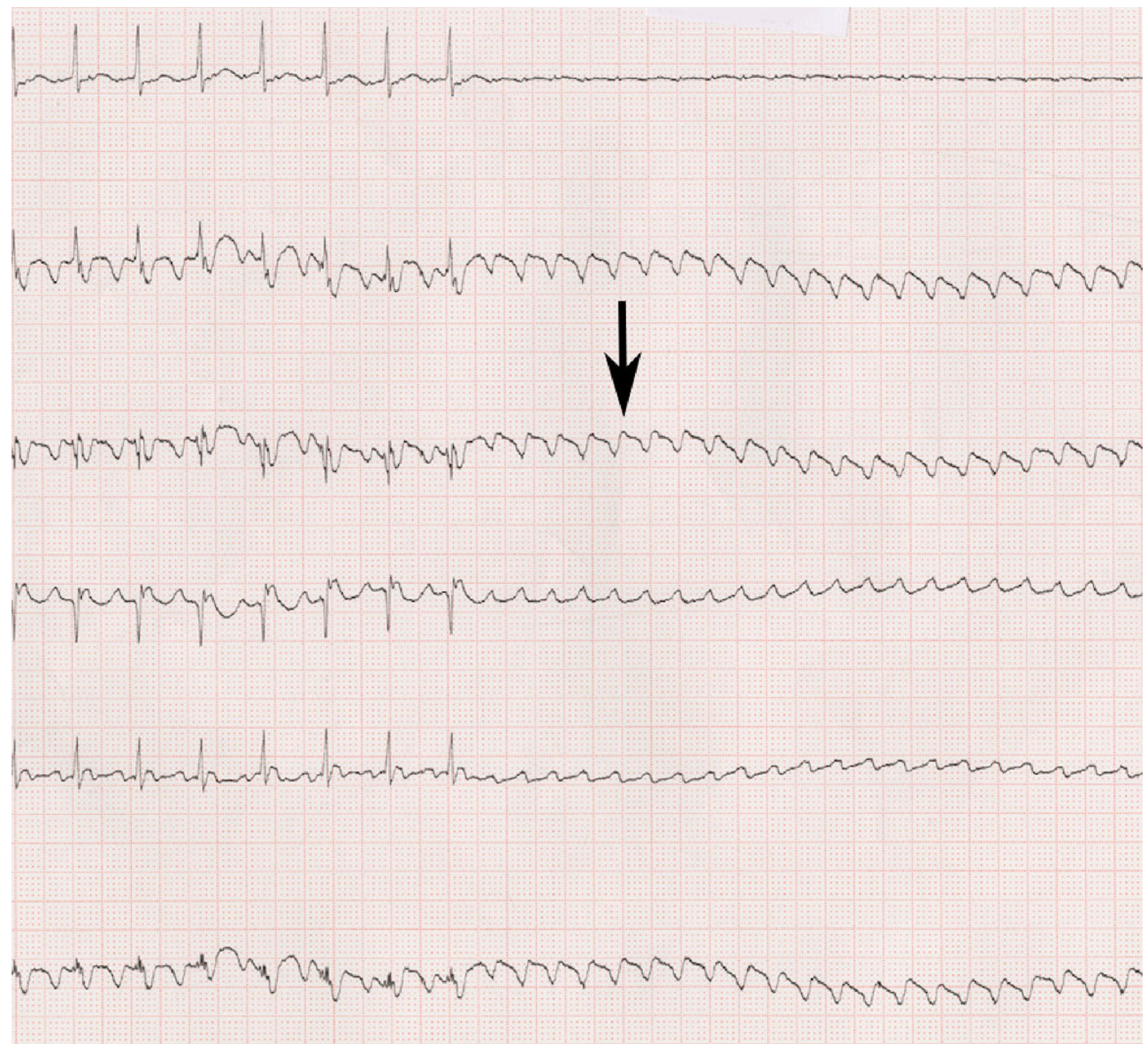

Fig. 5 ECG montrant des ondes F de flutter atrial (flèche) révélées par un massage du sinus carotidien D’après Pasquier et al. [6] (Copyright (C) 2017 Massachusetts Medical Society. Reprinted with permission)

\section{Conclusion}

Le MSC est fréquemment pratiqué au chevet du patient pour le diagnostic des syncopes ainsi que pour l'évaluation diagnostique ou le traitement de certaines tachycardies. Une évaluation minutieuse avant la procédure est nécessaire afin de s'assurer qu'il n'existe pas de contre-indication au massage. Le patient doit être préparé convenablement et monitoré durant la procédure. Le médecin doit être conscient des complications rares mais potentiellement graves et être capable, le cas échéant, de les prendre en charge.

Remerciements Les auteurs remercient le Dr Marc Andronikof, chef du service des urgences adultes du CHU Antoine-Béclère (Assistance publique-hôpitaux de Paris, Clamart, France), pour sa relecture attentive du manuscrit

Liens d'intérêts : les auteurs déclarent ne pas avoir de lien d'intérêt.

\section{Références}

1. Hering HE (1927) Die Karotissinusreflexe auf Herz und Gefäße vom normal-physiologischen, pathologisch-physiologischen und klinischen Standpunkt. Dresden und Leipzig Verlag von Theodore Steinkopf, Dresde

2. Weiss S, Baker JP (1933) The carotid sinus reflex in health and disease: its role in the causation of fainting and convulsion. Medicine (Baltimore) 12:297-354

3. Schweitzer P, Teichholz LE (1985) Carotid sinus massage: its diagnostic and therapeutic value in arrhythmias. Am J Med 78:645-54

4. Wieling W, Krediet CTP, Solari D, et al (2013) At the heart of the arterial baroreflex: a physiological basis for a new classification of carotid sinus hypersensitivity. J Intern Med 273:345-58

5. Brignole M, Moya A, de Lange FJ, et al (2018) ESC guidelines for the diagnosis and management of syncope. Eur Heart $\mathrm{J}$ 39:1883-948

6. Pasquier M, Clair M, Pruvot E, et al (2017) Carotid sinus massage. N Engl J Med 377:e21

7. Epstein AE, DiMarco JP, Ellenbogen KA, et al (2013) ACCF/ AHA/HRS focused update incorporated into the ACCF/AHA/ HRS 2008 guidelines for device-based therapy of cardiac rhythm abnormalities: a report of the American College of Cardiology 
Foundation/American Heart Association Task Force on Practice Guidelines and the Heart Rhythm Society. J Am Coll Cardiol 61:e6-75

8. Krediet CT, Parry SW, Jardine DL, et al (2011) The history of diagnosing carotid sinus hypersensitivity: why are the current criteria too sensitive? Europace 13:14-22

9. Waxman MB, Wald RW, Sharma AD, et al (1980) Vagal techniques for termination of paroxysmal supraventricular tachycardia. Am J Cardiol 46:655-64

10. Parry SW, Richardson D, O'Shea D, et al (2000) Diagnosis of carotid sinus hypersensitivity in older adults: carotid sinus massage in the upright position is essential. Heart 83:22-23

11. Brignole M, Alboni P, Benditt DG, et al (2004) Guidelines on management (diagnosis and treatment) of syncope - update 2004, Aerospace 6:467-537

12. Brignole M, Auricchio A, Baron-Esquivias G, et al (2013) ESC guidelines on cardiac pacing and cardiac resynchronization the- rapy: the Task Force on Cardiac Pacing and Resynchronization Therapy of the European Society of Cardiology (ESC): developed in collaboration with the European Heart Rhythm Association (EHRA). Europace 15:1070-118

13. Appelboam A, Reuben A, Mann C, et al (2015) Postural modification to the standard Valsalva manoeuvre for emergency treatment of supraventricular tachycardias (REVERT): a randomised controlled trial. Lancet 396:1747-53

14. Munro NC, McIntosh S, Lawson J, et al (1994) Incidence of complications after carotid sinus massage in older patients with syncope. J Am Geriatr Soc 42:1248-51

15. Puggioni E, Guiducci V, Brignole M, et al (2002) Results and complications of the carotid sinus massage performed according to the "method of symptoms." Am J Cardiol 89:599-601

16. Davies AJ, Kenny RA (1998) Frequency of neurologic complications following carotid sinus massage. Am J Cardiol 81:1256-7 